\title{
Spontaneous atrial fibrillation in a freestyle skier
}

\author{
G Whyte, N Stephens, S Sharma, R Shave, R Budgett, W J McKenna
}

Br J Sports Med 2004;38:230-232. doi: 10.1136/bjsm.2003.006197

A male freestyle skier was found to have atrial fibrillation during a routine physiological assessment. This was found to be associated with the consumption of an unusually large amount of alcohol. Athletes should be counselled about the potential dangers of alcohol consumption before exhaustive exercise.

\section{CASE REPORT}

In October 2002, a 19 year old white male freestyle skier presented to the British Olympic Medical Centre for routine physiological assessment. After the completion of a standard questionnaire and an informed consent form, it was established that the athlete had no medical history of note, including no family history of heart disease, and he was a non-smoker, took occasional alcohol, and was not taking any drugs at the time of investigation.

Routine physiological assessment included an incremental treadmill test to volitional exhaustion to ascertain $\dot{V}_{2}$ MAX, during which expired gases were collected at the mouth (Oxycon Alpha; Viasys, Brighton, Sussex, UK), and heart rate was monitored using telemetry (Polar Electro, Oy, Finland). After the test, the athlete's heart rate failed to fall as expected. Further, he complained of chest pain and shortness of breath. He was consequently attached to a 12 lead electrocardiograph (ECG), which revealed atrial fibrillation with a ventricular rate of 155 beats/min (fig 1). The athlete was monitored for two hours, during which time the atrial fibrillation persisted. He became mildly compromised, indicated by a fall in blood pressure and worsening discomfort associated with chest pain and shortness of breath. After an echocardiographic interrogation to determine the absence of structural heart disease and normal cardiac function, he was admitted to the coronary care unit. ${ }^{1}$ The atrial fibrillation was successfully cardioverted with flecanaide (150 mg over 30 minutes), and after 24 hours of observation he was discharged.

On follow up examination four weeks later, the athlete was in sinus rhythm, with normal venous pulse and normal heart sounds. Resting blood pressure was 124/80 mm Hg. Echocardiography showed normal intracardiac dimensions with normal systolic function of both left and right ventricles, and a 24 hour ECG showed sinus rhythm throughout. During integrated cardiopulmonary exercise stress testing, the athlete completed one minute and 20 seconds of level 5 of a BRUCE protocol stopping when maximal exercise capacity was reached. He achieved a $\dot{\mathrm{V}}_{2} \mathrm{MAX}$ of $53.5 \mathrm{ml} / \mathrm{kg} / \mathrm{min}(150 \%$ of age and sex predicted) and a maximum heart rate of 201 beats/min (100\% of age predicted). Blood pressure and heart rate response during exercise was normal. There were no inducible ECG changes or arrhythmias during or after exercise.

During consultation the athlete admitted consuming 12 units of alcohol, which was an excessive amount compared with his normal consumption, two days before the initial physiological assessment. The observed atrial fibrillation was diagnosed as a lone episode of alcohol induced atrial fibrillation and the athlete was counselled on alcohol consumption and vigorous exercise. (The term lone atrial fibrillation is used to describe an isolated occurrence and generally applies to young patients without clinical or echocardiographic evidence of cardiopulmonary disease.) No further action was taken and the athlete has reported no further occurrence of symptoms indicative of atrial fibrillation.

\section{DISCUSSION}

In the absence of an ECG before exercise and ECG monitoring throughout the exercise test, it is difficult to ascertain if the observed atrial fibrillation was present before exercise. The athlete, however, was symptomless with a heart rate of about 70 beats/min at the onset of exercise. It is therefore likely that the observed atrial fibrillation represented the first presentation of the arrhythmia. Atrial fibrillation may have a number of acute temporary causes, including drug use, ${ }^{2}$ surgery, electrocution, myocardial infarction, pericarditis, myocarditis, pulmonary embolism or other pulmonary diseases, and hyperthyroidism or other metabolic disorders. ${ }^{3}$ Most pertinent to this case study, however, is the effect of alcohol on the presentation of atrial fibrillation. Often termed "holiday heart syndrome", alcohol may increase the irritability of the atria resulting in the potential for catecholamine induced atrial fibrillation associated with exhaustive exercise.

Supraventricular arrhythmias are uncommon in athletes, except for atrial fibrillation which may occur more often than in the general population because of a high vagal tone. ${ }^{4}$ Vagally mediated atrial fibrillation appears to be more prevalent in male athletes ${ }^{5}$ and is often associated with lone atrial fibrillation. ${ }^{3}$ In the absence of structural cardiac disease, the likely cause of atrial fibrillation is atrial tachycardia originating at the insertion of the pulmonary veins into the left atrium which degenerates into atrial fibrillation. ${ }^{6}$ The ventricular response to atrial fibrillation depends on the electrophysiological properties of the AV node and the level of vagal and sympathetic tone. Extremely rapid ventricular rates ( $>200$ beats/min) suggest the presence of an accessory pathway. The athlete in this case study did not have an accessory pathway, as evidenced by a ventricular rate of 155 beats/min, together with the normal PR interval and absence of delta waves on the resting ECG after cardioversion (fig 2). Analysis of the resting ECG reveals a QS pattern in VI indicative of incomplete right bundle branch block, together with repolarisation abnormalities, including ST segment elevation and peaked $\mathrm{T}$ waves. These findings are common in athletes and represent a physiological adaptation to training. ${ }^{7}$

In athletes presenting with self limiting episodes of atrial fibrillation, the incidence of thromboembolism is very low, ${ }^{4}$ and antiarrhythmic drugs to prevent recurrence 


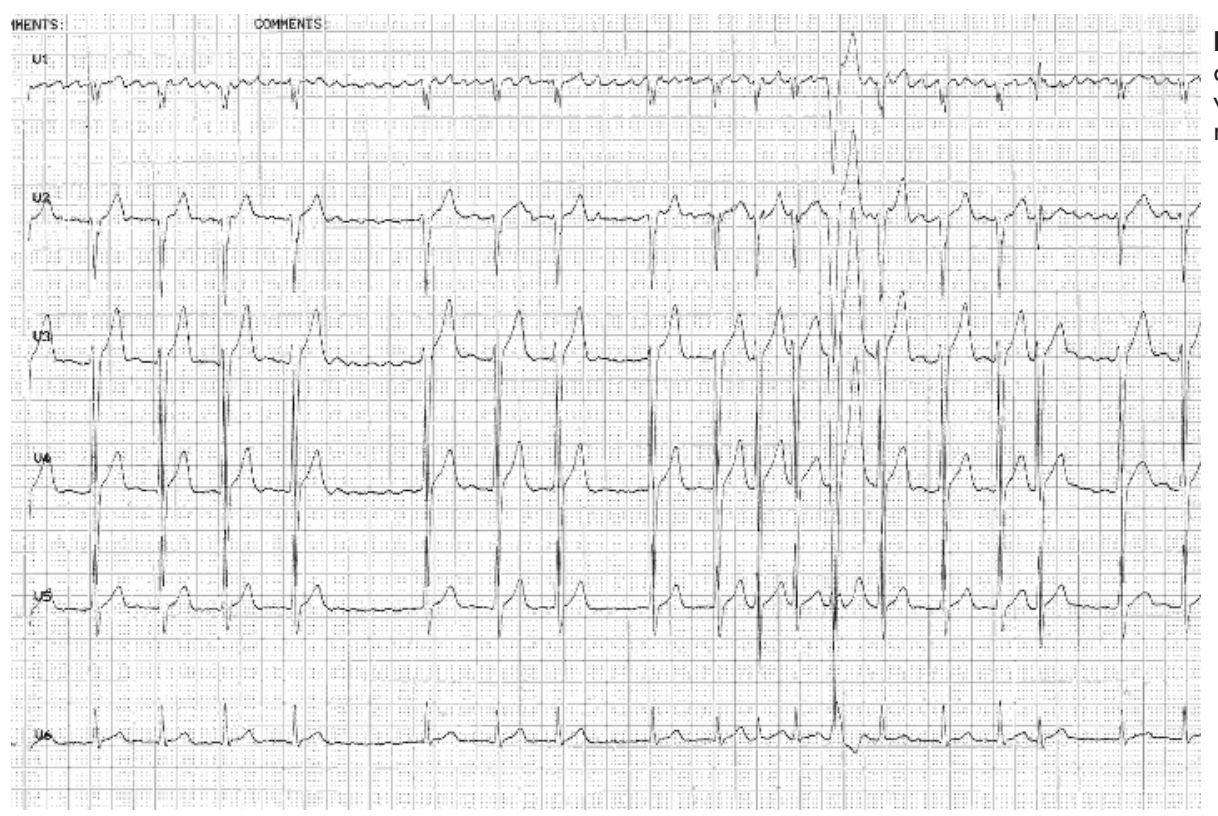

Figure 1 Electrocardiogram obtained after exercise showing atrial fibrillation with a ventricular rate of 155 beats/ $\min$.

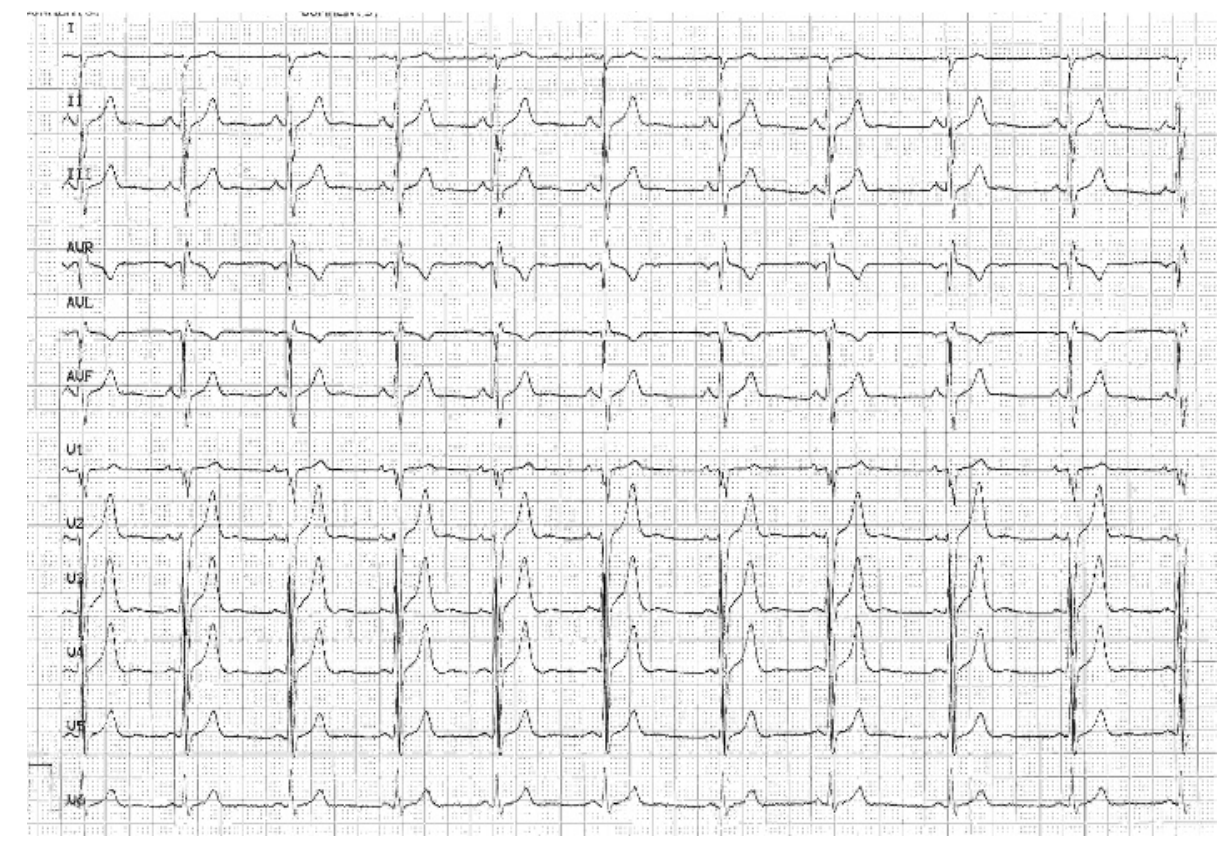

Figure 2 Resting electrocardiogram at four weeks follow up, after cardioversion.

\section{Take home message}

Small volumes of alcohol may represent a relatively large amount in an elite athlete. Athletes should be counselled about the potential dangers of the consumption of unusually large quantities of alcohol before exhaustive exercise. ECG monitoring during physiological assessment is crucial to improve the care and safety of the athlete, and should be adopted as standard. Although atrial fibrillation is more prevalent in athletes, its appearance remains uncommon. Care must be taken in the interrogation of the ancestry of an athlete presenting with atrial fibrillation to eliminate the possibility of familial atrial fibrillation. are usually unnecessary, unless atrial fibrillation is associated with severe symptoms and/or structural heart disease. $^{3}$ For those athletes with symptoms, $\beta$ blockers or calcium channel blockers may provide some relief. However, $\beta$ blockers are currently on the banned drug list for many sports. The use of RF ablation of the atrial tachycardia focus may be warranted for those athletes with recurrent or troublesome episodes of paroxysmal atrial fibrillation.

Cardiac arrhythmias in athletes range from the benign and asymptomatic to the symptomatic and potentially life threatening. ${ }^{4}$ The high vagal tone associated with physical training may result in athletes being more susceptible to certain arrhythmias including atrial fibrillation. It is impor- 
tant to determine which athletes with arrhythmia require follow up and treatment, and those that can safely return to training and competition.

\section{Authors' affiliations}

G Whyte, N Stephens, S Sharma, R Shave, R Budgett, W J McKenna, CRY Centre for Sports Cardiology, British Olympic Medical Centre, Northwick Park Hospital, Harrow, Middlesex, UK

Correspondence to: Dr Whyte, CRY Centre for Sports Cardiology, British Olympic Medical Centre, Northwick Park Hospital, Harrow, Middlesex HAl 3UJ, UK; greg.whyłe@boa.org.uk

Accepted 24 June 2003

\section{REFERENCES}

1 Nolan J, Baskett P, Gabbott D, et al. Advanced life support manual. 4th ed. London: Resuscitation Council (UK) and European Resuscitation Council, 2000.

2 Sullivan M, Martinez C, Gallagher E. Atrial fibrillation and anabolic steroids. $J$ Emerg Med 1999;17:851-7.

3 ACC/AHA/ESC. Guidelines for the management of patients with atrial fibrillation. Eur Heart J 2001:22:1852-923.

4 Link M, Homoud M, Wang P, et al. Cardiac arrhythmias in the athlete. Cardiol $\operatorname{Rev} 2001 ; 9: 21-30$

5 Furlanello $F$, Bertoldi A, Dallago M. Atrial fibrillation in elite athletes. $J$ Cardiovasc Electrophysiol 1998;9:S63-8.

6 Haissaguerre M, Jais P, Shah D. Spontaneous initiation of of atrial fibrillation by ectopic beats originating in the pulmonary veins. N Engl J Med 1998;339:659-66.

7 Sharma S, Whyte G, Elliott PM, et al. Electrocardiographic changes in 1000 highly trained elite athletes. $\mathrm{Br}$ J Sports Med 1999;30:319-24. 